\title{
La scrittura di Volponi tra natura e storia. Ideologia ed eros in Il lanciatore di giavellotto
}

Alfredo Luzi

\section{OpenEdition}

\section{Journals}

Edizione digitale

URL: http://journals.openedition.org/cei/281

DOI: $10.4000 /$ cei.281

ISSN: 2260-779X

Editore

UGA Éditions/Université Grenoble Alpes

\section{Edizione cartacea}

Data di pubblicazione: 15 juin 2005

Paginazione: 139-153

ISBN: 978-2-84310-066-6

ISSN: 1770-9571

Notizia bibliografica digitale

Alfredo Luzi, «La scrittura di Volponi tra natura e storia. Ideologia ed eros in I/ lanciatore di giavellotto», Cahiers d'études italiennes [Online], 3 | 2005, online dal 15 décembre 2006, consultato il 27 mars 2021 URL: http://journals.openedition.org/cei/281 ; DOI: https://doi.org/10.4000/cei.281 


\title{
LA SCRITTURA DI VOLPONI TRA NATURA E STORIA
}

\author{
IDEOLOGIA ED EROS IN IL LANCIATORE DI GIAVELLOTTO
}

\author{
Alfredo Luzi \\ Università di Macerata
}

La scrittura di Volponi presenta una costante contiguità tra poesia e narrativa, evidente fin dalle prime opere poetiche come Il Ramarro (1948) e ancora riscontrabile nelle pagine dell'ultimo romanzo La strada per Roma (1991). Essa testimonia la crisi di un linguaggio novecentesco, ormai esausto dopo l'esperienza ermetica e sempre più ambiguo, vischioso, torbido nella sua densità semiotica. Ne è conferma l'adozione di titoli la cui polisemia è accentuata dall'uscita attributiva in -ale, così frequente nelle opere dell'urbinate (Memoriale, La macchina mondiale, Corporale, Sipario ducale, Foglia mortale, Le mosche del capitale, Nel silenzio campale). E se in Memoriale si intreccia il senso di una biografia ricostruita sul filo della memoria con quello di un documento-denuncia trascritto da chi si sente sotto processo, nella Macchina mondiale l'idea del congegno convive con quella, di estrazione seicentesca, di un progetto cosmico evidenziato da una rappresentazione teatrale. In Corporale il riferimento a tutto ciò che è organico, che appartiene alla fisicità animale, si depura nella sacralità mistica che il lemma simbolizza nella liturgia cattolica, mentre nell'aggettivo campale convergono i concetti di spazialità, figuralità e conflittualità. Nell'insieme dei titoli si può, d'altro canto, leggere la configurazione di una sequenza ritmico-musicale.

$\mathrm{Ma}$, a livello gnoseologico, l'unità tra dimensione lirica e dimensione prosastica è anche il segno del tentativo di realizzare la simbiosi tra natura e storia, un rapporto categoriale elaborato in particolare dalla filosofia romantica e utilizzato da Volponi già nell'Antica moneta (1955), dove il soggetto si presenta non come io assoluto ma come groviglio, tendente ad una forma di conoscenza più comprensiva che selettiva. Nel rapporto con la natura Volponi conferma la sua adesione al mito della madre terra e la compartecipazione alla naturalità, proponendo, sul piano ermeneutico, 
una connessione inscindibile tra soma e pneuma. Il primo strumento di conoscenza è dunque il sentire, che permette all'uomo di percepire il movimento di sistole e diastole della natura, di apertura e di chiusura, di vita e morte, lo spazio entro cui è possibile rintracciare le impronte del caos primigenio. Il viaggio nel corporale è nello stesso tempo una nostalgia del reditus ad uterum, attraverso la simbologia della donna, e un desiderio di vivere nella storia, definita dalla progressione temporale che trama in profondo le prime opere dell'autore.

Ne consegue una struttura ossimorica della scrittura, da una parte tendente alla narrazione autobiografica, in cui predomina il controllo dell'io, dall'altra comprensiva del paradigma ambivalente dell'essere e del nulla che viene diacronicamente riaffermato nell'esistenza collettiva. La funzione gnoseologica del sentire è così rafforzata dalla capacità proiettiva dello sguardo, in grado di far luce " dentro » l'io ma anche " fuori » di esso, in un continuo gioco di rapporti tra oggetto e soggetto. Atto percettivo che porta alla conoscenza del mondo attraverso la messa in luce delle divergenze esistenti tra la molteplicità analitica e frazionata del mondo naturale e l'unità complessiva, anatomica, dell'io, il guardare equivale, già nell'Antica moneta, non solo a constatare gli eventi ma a dare loro un senso, un'unità, in modo che all'interno del soggetto si ricostituisca quell'armonia tra io e cosmo, tra pensiero e visione, che è alla base della cultura antropologica volponiana. Questo andare a caccia di immagini, di eventi, di epifanie, questo indagare nei minimi elementi che compongono ed animano il paesaggio, corrisponde al modello, nella cultura popolare, dell'uomo che si proietta nella storia, è l'anticipazione e il rovescio di quell'" acontistés, che vuol dire anche lanciatore di dardi, lanciatore di sguardi, lanciatore di desideri... lanciatore di se stesso ${ }^{1}$ ". Seguendo modalità epistemologiche che si avvicinano all'idea di Lebenswelt (mondo della vita) di Husserl, l'io del poeta affida ad un $t u$, che non è più e non solo il suo alter-ego ma la proiezione sintetica e simbolica della collettività, il compito di dare un senso unitario allo svolgersi dell'esistenza, al succedersi di azioni in apparenza disaggregate ed invece coordinate in un rapporto armonico con la grande madre natura.

La speranza di uno sviluppo individuale e collettivo armonico, dal giardino dell'adolescenza al mondo felice adulto, si configura, nelle Porte dell'Appennino (1960), come il germe della linea utopica che attraversa indenne le opposizioni città-campagna, uomo-paesaggio, dentro-fuori, 
che costituiscono la struttura tematica del volume. E indubbiamente, almeno a livello ideologico, questa persistenza della coscienza del possibile, sottolineata nella scrittura come alternativa alla paura di vivere la realtà, trova la sua elaborazione nella esperienza di "Officina ", un laboratorio letterario e culturale in cui da una parte si recupera attraverso lo studio della tradizione lirica europea il valore simbolico della parola e dall'altra si teorizza sulla efficacia della proposta gramsciana di fondere, attraverso la figura dell'intellettuale organico, sentire e sapere.

La connessione antinomica persiste nei romanzi, a partire da Memoriale (1962), dove la difesa della soggettività è tutta accentrata nel rifiuto della differenziazione illuministica tra follia e ragione e nella lotta tra autenticità della natura e falsità dei rapporti sociali. Sicché è possibile individuare fin dal primo romanzo una sorta di macrosequenza filosofico-concettuale che, con diverse modalità, si ripete nello schema gnoseologico volponiano : l'io si rapporta con il paesaggio umano, entro il cui spazio consuma la sua vicenda esistenziale, fatta di relazioni, più o meno conflittuali, con la società, producendo così storia ma nello stesso tempo definendo la sua personalità di base.

In Memoriale Albino Saluggia si colloca al centro della contraddizione tra la tendenza alla reificazione insita nell'esaltazione del lavoro in fabbrica, secondo la teoria del capitalismo avanzato, e la nostalgia per un mondo, idillico e georgico, che sta scomparendo ma che egli ha interiorizzato e alle cui immagini ricorre quando desidera difendersi dagli attacchi della società, tutelando la propria diversità. Albino è, in fondo, uno dei capri espiatori volponiani : assumendo su di sé il groviglio dei sintomi del disagio sociale (e in questa prospettiva Volponi trasforma il tema della malattia da condizione fisiologica a condizione sociologica), lotta per il trionfo dell'utopia. Egli, che ha conosciuto un passato di prigionia, si sente ora di nuovo prigioniero nel mondo della fabbrica, un mostro incontrollabile e potente che traumatizza continuamente la coscienza naturale di Albino. Il tema oppositivo città/campagna, presente in molta parte della letteratura italiana del '900, viene così ripreso nella dimensione alienante del lavoro. L'aspirante ad « essere incluso nella quota reduci del contingente di manodopera da avviare al lavoro nella grande industria di $\mathrm{X}^{2}$ " rimane deluso e impaurito dalla fabbrica : "La fabbrica mi sembrava un edificio senza senso e sentivo che una parte del mio cervello stava facendo violenza su di me per trattenermi in quel luogo ostile e innaturale » 
(ibid., p. 23). La sua salvezza è nel ritorno alla campagna, al lago, ma senza speranza di aiuto :

Al bivio sono sceso, dopo un viaggio veloce, e a piedi mi sono diretto verso casa mia. Guardavo, come sempre, il lago crescere a poco a poco sotto i miei occhi, nella salita verso casa mia. A un certo punto era completamente sotto di me, che respirava piano tra le sue sponde. E così sotto di me tutti i tetti del paese, rossi e ordinati come se non albergassero la cattiveria umana. (ibid., pp. 307-308)

Giustamente Pasolini ha parlato, per questo romanzo, di due strati di linguaggio, quello del paranoico determinato dalla ossessività esistenziale (storia) e quello del poeta che contempla il paesaggio (natura). Ma sul piano strettamente stilistico è significativo il fatto che già in Memoriale, in particolare nell'ultima parte, sia presente quella particolare tecnica fonetico-generativa del linguaggio che caratterizzerà le opere successive di Volponi e che è il segno di una scrittura inclusiva, che rifiuta la divisione dei generi e tende invece alla condensazione di significante e significato. Saluggia-Volponi spiega così quel rampollare delle parole dentro il suo essere :

Ecco, andavo dietro alle parole : il loro suono contava più di ogni altra cosa, più del loro senso, ed io finivo per ordinarle o per trovarle o per inventarle secondo il suono, senza più l'ordine del significato e del pensiero. Ma così trovavo un altro ordine pieno di emozioni e che parlava meglio il mio linguaggio. Non andavo nemmeno più dal prete perché anche la mia anima si apriva ormai sopra di me. Seguivo i miei discorsi immobile, con la mente, anche se gustavo le parole tra le labbra e i denti, pronunciandole nelle ripetizioni e in tutte le rime, come dolci catene. Inventavo e cantavo le litanie dei miei dolori e della mia vittoria. Certi giorni mi veniva in mente, al posto delle parole, un motivo musicale o un ritornello e allora lo seguivo per tante ore, ondeggiante come un aquilone e il suo filo si svolgeva nella mia mente e trascinava in volo i miei pensieri che si staccavano senza farmi male, partendo dalla mia testa, continuando nell'aria la circolazione del sangue leggiero della mia testa, senza strappare nulla dal mio cuore, dal centro di me. (ibid., p. 284)

Pochi sanno che il pre-testo (nel significato più ampio del termine) de La macchina mondiale (1965) ha la stessa derivazione di interesse fonologico e linguistico, per di più individuata nell'esperienza storica ed esistenziale di un contadino marchigiano, Pietro Mario Vallasciani, semianalfabeta ma innamorato del mistero delle parole, compilatore di trattati scientifico-utopici, incontrato dallo scrittore urbinate ad Ivrea. Carlo Benedetti, partendo dalla avvertenza premessa da Volponi al romanzo, in cui si conferma che " $\mathrm{i}$ riferimenti alla realtà storico-sociale sono molteplici ${ }^{3}$ " e che "le idee del protagonista sulla genesi e sulla palingenesi derivano da quelle che il signor P. M. V. sta svolgendo e sistemando, insieme con altre, in un trattato "Per la costituzione di una nuova Accademia

3. La macchina mondiale, Milano, Garzanti, 1965, p. 5. 
dell'Amicizia di qualificato popolo» (ibid., p. 5), ha ricostruito, in un gustoso e documentato articolo su "Liberazione ", la vicenda di questo militante comunista, convinto sostenitore della idea che " le parole nascono e si riproducono » e inconsapevole produttore di utopia. In effetti, l'Anteo Crocioni, personaggio centrale del romanzo, ha molto della figura del Vallasciani. Giovane contadino marchigiano degli anni cinquanta, egli non è né matto né asociale : semmai può considerarsi un filosofo utopista, disintegratore del sistema sociale in vigore e teorico visionario di un futuro armonioso di fraternità umana. Ma proprio per questa sua diversità egli è rifiutato dalla società borghese, incapace di comprendere una razionalità non conservatrice, ma proiettiva, che tende a definire le spinte della creatività. Crocioni, progettando il suo suicidio con una esplosione che disintegri l'ordine costituito dalla collettività, desidera ribellarsi alla integrazione massificante della società contemporanea. La sua invocazione-invettiva alla propria terra è da considerare un innesto della tematica della ribellione e della sconfitta all'interno di un progetto rivoluzionario. Anteo non è pazzo; pazzi sono gli altri, incapaci di avere fiducia nella fantasia, nel possibile, nella utopia :

"Ah, Marche, » dissi, « ah, terra marchigiana, io so anche come la tua pazzia può essere ingrata e so come non ci si debba fidare della tua natura. Mi metterò dunque io adesso ad esaltare la tua bellezza perversa, la fissità crudele della tua natura ? Io sono sempre Anteo e torno per lavorare dietro i miei pensieri e non per cedere ai tuoi frutti ; io debbo costruire altri frutti e liberare gli uomini e anche la loro campagna, dalla morte, dalle inutili giornate che si passano a stare a guardare la campagna e la natura e ad aspettare la morte senza fare niente che possa diventare meglio della morte stessa " (ibid., p. 198)

Anteo, anche lui dilaniato tra vita e morte, tra natura e coscienza, affida alla progettazione del proprio trattato la funzione di recupero dell'euforico rapporto tra uomo e terra. $\mathrm{E}$ in quest'ambito è ribadita la circolarità sintetica del sapere che, pur utilizzando linguaggi diversi, mira ad una complessiva gramsciana acquisizione di coscienza, un tema, come si vede, che in Volponi assume il carattere di ossessività gnoseologica :

Invece oggi posso dire che il sentire poetico, che è la visione artistica, è anch'esso uno strumento della scienza; oppure che la scienza può avere due teste come ventimila o due milioni, e penso che la felicità delle macchine libere sia una felicità artistica, che si gode nello stesso momento in cui progredisce per l'intervento di chi la fa progredire. In quello stesso momento in cui vive e progredisce, in cui uno fa e gode come nelle creazioni. (ibid., p. 274)

Un testo fondamentale per definire, anche sul piano stilistico, la poetica volponiana dello spazio e del tempo, della natura e della storia è Foglia mortale, un volumetto, pubblicato in tiratura limitata nel 1966, che raccoglie cinque componimenti in cui è evidente lo sforzo dello scrittore di adeguare il processo di analisi psicologica alla ricerca linguistica e formale. Dal paesaggio, spazio aperto e invitante, giunge all'io un segno che deter- 
mina una inquietudine e un disagio interni al soggetto, e che, a loro volta, si riproiettano nello spazio visivo, stravolgendolo e rendendolo minaccioso alla coscienza del soggetto. Ma, pian piano, proprio attraverso l'andamento poematico e l'accumulo di temi e suggestioni, il linguaggio assume una funzione orientante fino a ristabilire una situazione psicologica di equilibrio tra natura e coscienza. Utilizzando le opposizioni come tracce delle intime contraddizioni Volponi colloca al centro della propria problematica gnoseologica il superamento della frontiera, del diaframma, tra natura e storia, fra individuo e società. Immerso nel labirinto del reale, consapevole dei limiti di una lingua oscillante tra la germinazione verbale e nominale (una sorta di monologo interiore del subconscio) e la potenzialità definitoria (la lingua che predica l'essere), egli difende la centralità dell'uomo, la sua aspirazione alla globalità e complessità dell'esperienza :

Allora il paesaggio è una vita quieta dove/l'uomo entra e lavora e guarda e muove,/- può ribaltarsi e girare - e muove fino alla estrema/ultima riga, alla estrema freddezza della sua coscienza ;/ allora la natura è la figura, l'involucro o la serva di tale/coscienza. (La pretesa d'amore) ${ }^{4}$

Nel 1974 Volponi pubblica Corporale.

Nel romanzo quelle spinte alla totalità e alla circolarità che erano già presenti nelle opere precedenti, inserite però in una scrittura narrativa rigida e dunque prive di potenzialità implosive, acquistano una forte carica destrutturante e determinano un profondo mutamento del modello romanzesco tradizionale. Volponi sconvolge le categorie cronotopiche, proponendo un percorso narrativo a spirale, avvolgente, chiuso su se stesso, ma sempre messo in gioco dalla dinamica della dislocazione fantastica. I personaggi non hanno unitarietà, anzi si presentano raddoppiati, o sdoppiati, nel loro contrario. Ne è un indizio stilistico l'uso alternato della prima, della seconda e della terza persona per indicare un unico protagonista. L'aspirazione all'onnicomprensività è rivelata dall'andamento magmatico del racconto, da quella deriva verso il non finito che è appena bilanciata da uno schema di riferimento, sempre sullo sfondo, a cui lo scrittore attribuisce la funzione di controllo razionale e concettuale del cumulo di parole con le quali egli cerca di penetrare e possedere la realtà.

Anche sul piano per così dire politico Corporale rappresenta il momento di frattura tra il prevalere del concetto di consumo ideologico negli anni sessanta e la delusione che alla vigilia del '68 porterà, per compensazione, al ritorno dell'utopia, dell'immaginazione al potere.

4. Foglia mortale, Ancona, Bucciarelli, 1974, pp. 48-49. 
Il protagonista di Corporale, Gerolamo Aspri, vive in un mondo 'disertato dalle favole', che invece con la loro permanenza nell'immaginario mitico e simbolico degli uomini avevano reso tollerabile l'esistenza. Egli soffre lo strappo traumatico da una civiltà, come quella contadina e mediterranea, che sta per morire, mentre attorno si leggono i segni della possibile esplosione di una bomba atomica, simbolo di una società che scoppia, che incombe come una minaccia sul futuro dell'umanità. È per questo che Gerolamo sente il bisogno di recuperare il rapporto con la terra, di rinchiudersi in essa, cercando senza sosta l'arcatana in cui occultare e preservare la propria intelligenza utopica. Anche questa volta, innestato più sul piano strutturale che sulla composizione dei personaggi, il rapporto tra razionalità e istinto, tra fisicità e procedimenti gnoseologici, è calato nel gioco dell'utopia, rappresentata, a livello strutturale e stilistico, da una geometria di progetti alternativi alla opacità reattiva del corpo biologico :

Anche nei giorni seguenti, davanti a qualche vento sabbioso o a qualche rumore che qui nasce davvero improvvisamente, tanto in città quanto nelle campagne intorno, mi sono sentito come se non avessi mai cercato niente altro che queste cose : come se non avessi mai cercato un'affermazione, ma davvero solo queste cose : una città favolosa, un frutteto, strade, alberi, acque, nebbie e questa legge del mio corpo che vuole sopravvivere, che ha deciso di rifiutare una regola mortale, affidandosi ad un suo particolare disegno che sta nel quadro generale similmente a uno di quei frutti del frutteto ; di questo mio corpo, che è più gentile e fortificato ${ }^{5}$.

Nel romanzo, il punto goniometrico, il riferimento costante di un teorema dimostrato con lucida follia tra un delirio letterario e un sogno erotico, è la città di Urbino. In un paesaggio che non si discosta se non incidentalmente e per ragioni strettamente strutturali dall'area di influenza attiva e passiva dell'ambiente montefeltresco, Urbino appare come ricettacolo della speranza di vita, scoperto dopo un viaggio a Pennabilli, il paese di Ivana, della donna della giovinezza e del desiderio. È la costante di un sistema antinomico entro cui si dibatte la psicologia del protagonista incerto nel subire il richiamo della propria sensibilità, la reattività corporale o il fascino di una lucida razionalità utopistica sempre attenta a rifiutare gli stimoli di una emotività incontrollata. Il ritorno alla terra madre oscilla fra la certezza di una salute animale ("Confermò che in ogni caso Urbino mi sarebbe stata salutare e che mi avrebbe giovato » [ibid., p. 101]) e la scoperta di una immobilità spaziale e temporale ("Girai per Urbino e ne rivelai chiaro ogni limite » [ibid., p. 105]). La città è nello stesso tempo il nido caldo, organico e vitale ( GGuardo e penso a una comunità biologica stretta, dove tutti partecipano degli stessi sentimenti e dello stesso cibo.

5. Corporale, Torino, Einaudi, 1974, p. 386. 
Ho l'impressione, dopo qualche passo, di essere dentro una noce : concentriche le costruzioni e i vicoli, oppure dentro un organo animale " [ibid., p. 277]) e il castello di fate ingannevole, edificato sul vuoto dell'immaginazione ( Non è certo in Urbino, entro le mura, il luogo dove potrò rifugiarmi. Tutto è un castello di carte con i giardini pensili uno sopra l'altro, le volte cieche che rimbombano sulle cave e sui pozzi. Diventerebbero trappole...» [ibid., p. 301]).

Il romanzo è dunque anche nel rapporto tra Gerolamo e Urbino, nel colloquio ininterrotto di Edipo con la propria madre, nell'ansia costante di mantenere vitalità ad un cordone ombelicale che lega ancora due cellule che si alimentano a vicenda :

Tutto sarà in circoletti o sprofondato nei pozzi ; invece a me sembra che tutto comunichi e sia anzi la continuazione non solo fisica di un altro pezzo, ma proprio il completamento. Più salgo e più mi si delinea nella mente l'immagine di questa città come di un corpo o di un organo reciso di un corpo sotto un cielo lenzuolo che ne fissa continuamente l'immagine : la succhia, la compone e subito la muta. (ibid., p. 285)

Nelle opere successive la dinamica natura-storia si configura con modalità differenziate, spesso occultata dall'incalzare della trama e dalla definizione a tutto tondo dei personaggi, e tuttavia sempre persistente in sottofondo, quasi un retroterra tematico e stilistico su cui erigere edifici narrativi di prorompente originalità.

Nel Sipario ducale (1975), ad esempio, ancora personaggi "diversi " escono dal "sipario " di Urbino per proiettare il lettore che segue le loro vicende nell'Italia della fine degli anni sessanta, quelli delle bombe di Piazza Fontana, delle trame oscure che cercavano di minare le fondamenta della repubblica. La simbologia della malattia è qui amplificata a immagine "pestilenziale " della stessa storia d'Italia, dove si intrecciano le vicende parallele (sostenute da una struttura duplice del racconto) di Oddino e il suo sogno ducale e dell'anarchico Subissoni assertore della necessità di una palingenetica rivoluzione. Il pianeta irritabile (1978) può invece essere letto come una metafora narrativa della possibilità di passare dalla catastrofe all'utopia di un nuovo mondo, partendo da una dimensione apocalittica, da day-after, che segna però la necessità di ricostruire sulle rovine del passato le basi di una nuova società. Ma è nel Lanciatore di giavellotto (1981) che il principio delle opposizioni costitutive ritrova una sua centralità.

Damìn, il personaggio del romanzo Il lanciatore di giavellotto, pubblicato da Volponi nel 1981, è nello stesso tempo un campione nel lancio del giavellotto ma anche un suicida che, dopo aver ucciso la sorella, si getta da un ponte..., lanciatore di se stesso, appunto. 
Nel romanzo il principio delle opposizioni costitutive ritrova una sua centralità. Volponi narra la storia drammatica (ambientata tra 1920 e 1940, dunque in pieno fascismo) di una educazione sentimentale e sociale, che dà al volume il tipico carattere del Bildungsroman. Esso si sviluppa su una struttura antinomica di forte impronta metaforica. L'adolescente Damìn si muove tra paura dell'esterno e rifugio nell'interno, tra regressione e sviluppo, tra proiezione naturale verso l'alterità con la scoperta della donna e del sesso e processo d'interiorizzazione dell'immaginario attraverso la masturbazione.

In questa prospettiva, risulta illuminante il brano in cui Damìn affida alla proiezione di un film la funzione simbolica e metaforica di condensazione della sua realtà :

Guardò il film senza prendervi completamente parte : forse per la prima volta nella sua vita a un film di quel genere. La canzone del capo negro cantava più che la schiavitù e la giungla, il dolore suo e di sua madre : mischiava con quegli accenti stranieri e profondi, da negro, i suoi dolori e li congiungeva e li alzava in una nota sola, finché non li sperdeva tra i tamburi. C'era un fiume anche nel film; largo, disteso in mezzo agli alberi del pane, ma poi impetuoso e rotto da tante cascate che segnavano la fine del territorio della tribù. Il suo territorio domestico e fluviale era stato sempre segnato dall'ansa renosa del Metauro dopo il primo ponte di Fossombrone, là dove scendevano le carriole e i carri degli sbadilanti e dei cavatori ; tutta gente che non amava il fiume, secondo suo nonno, e che lo feriva con gli scavi, rovinandone le rive e le correnti....fino a che non sarebbero caduti anche i ponti, anche le mura... e tutta la città nella rena ${ }^{6}$.

E' l'adolescenza stessa di Damìn a produrre una predisposizione gnoseologica che ricorre prevalentemente all'icona come momento formatore del rapporto tra l'organismo e la realtà. E il disegno risulta la forma espressiva più funzionale allo scopo, molto più del racconto orale o della scrittura :

Disegnare gli consentiva di poter guardare ancora intorno a sé, di poter sopportare la realtà e anche di comprenderla e conservarla secondo una tendenza che non la mettesse subito e aspramente in conflitto con quella sua propria interna.

Il disegno restituiva le cose, i brani della giornata e del paesaggio al suo dominio, a una convinzione di se medesimo, in rapporto con le scene e oggetti, che non fosse subito e tutta dolorosa e che non producesse, con gli inciampi e le associazioni consueti, motivi nuovi di dolore o comunque la spinta al suo continuo dilagare. (ibid., p. 171)

Le tracce della visività sono le linee entro le quali il corpo scopre se stesso, proiettandosi verso l'esterno e mettendosi nel contempo in conflitto con esso :

Damìn si misurò di continuo le ginocchia e la cintura e restò per ore intere fermo davanti alla finestra con le mani strette ognuna sul polso dell'altra. Guardava le proprie scarpe e ne riconosceva la fisionomia, le linee distinte e convergenti presso la loro precisa identità ; dentro vi

6. Il lanciatore di giavellotto, op. cit., p. 137. 
erano i suoi piedi : in qualche modo gli erano sottratti e solo la sera dentro il letto poteva sentirli liberi e nudi, e per potere prenderne l'intero possesso iniziava a imbeccare l'uccello.

Dopo il suo corpo stava tutto insieme, completo e sigillato dall'appagamento e da un senso totale di colpa, che non doveva mai essere smosso da nessun stimolo e libertà, perché stabiliva per lui una condizione comune a quella della madre, con la medesima fonte e quantità d'ossigeno per continuare. (ibid., p. 149)

Si è dunque in presenza di quel meccanismo che Lacan ha chiamato "stadio dello specchio»:

Le stade du miroir est un drame dont la poussée interne se précipite de l'insuffisance à l'anticipation - et qui pour le sujet, pris au leurre de l'identification spatiale, machine les fantasmes qui se succèdent d'une image morcelée du corps à une forme que nous appellerons orthopédique de sa totalité - et à l'armure enfin assumée d'une identité aliénante, qui va marquer de sa structure rigide tout son développement mental $^{7}$.

La totalità primordiale è stata rotta dalla scissione del mito dell'ermafrodita, dal taglio dell'unità rappresentato, anche semioticamente, dal sesso, sentito come mistero verso cui si è attratti perché nell'alterità contiene i segni occulti dell'identità. Il contatto con il proprio sesso si trasforma allora in un meccanismo di presa di coscienza. La masturbazione assidua a cui si sottopone Damìn è insieme verifica della propria individualità, e scoperta della sofferenza come percezione indotta dal senso di colpa interna alla propria soggettività :

Dopo aver mangiato andò presto a dormire, con l'idea di mettersi a fare e a cuocere vasi appena finite le scuole e di diventare un libero pensatore, capace di agire da solo contro tutti e anche di lottare contro l'ingiustizia. Vagò nel dubbio se i propri dolori, progetti e inquietudini fossero o no da considerare idee e se quindi lui fosse o non fosse un idealista, destinato di conseguenza a diventare fascista e dirigente. Allora si strinse su se stesso e abbracciato al proprio uccello volò via $^{8}$.

All'onanismo come chiusura sulla propria identità corporea fa da pendant il voyeurismo come proiezione verso l'esterno, verso la scoperta della diversità sessuale della donna. Un po'come l'Agostino di Moravia, anche Damìn scopre che sua madre è una donna, spiandola mentre si spoglia, mentre copula con Marcacci, mentre pratica una fellatio al suo amante.

In un linguaggio in cui la linea normativa della lingua ufficiale, utilizzata con finalità esterne al soggetto e comunque collettive, è continuamente rotta dal ricorso al dialetto per fare emergere i grumi di visceralità e di passione dell'individuo, Volponi inserisce alcuni lacerti di germinazione verbale, frequenti nelle sue ultime composizioni poetiche, ma soprattutto sviluppa la macrometafora ossessiva del 'vaso'che si identifica con la vagina (il nonno di Damìn costruiva vasi, Damìn imparerà a far vasi) :

7. J. Lacan, Écrits I, Paris, Éditions du Seuil, 1966, pp. 93-94.

8. P. Volponi, Il lanciatore di giavellotto, op. cit., p. 60. 
Cosa insegnano l'Iliade e l'Odissea? Non tutti sono Achille o Agamennone, e nemmeno Aiace e nemmeno Ulisse o Ettore : qualcuno può essere Menelao. Tanti invece i marinai, gli arceri, i fanti, i portatori ; i portatori di provviste, di armi, di vasi.

"Vasi, - ripeté Damìn a se stesso, - pieni di tempo e di sciagura ». (ibid., p. 55)

Hai mai letto I miserabili di Victor Hugo, Oliver Twist di Dickens ? Oppure L'assommoir di Émile Zola o La madre di Gorkij ? Perché una volta non fai una gita a Pesaro a sentire La Traviata? Questa almeno l'ha scritta un italiano.

Esci dal tuo vaso, Damìn, che è ora.

Fa' come il tuo nonno che i vasi li fabbrica lui ; li fa lui come vuole e poi li manda via, li vende. Damìn era preso da un sentimento misto, che lo pungeva di pena e di rabbia alla parola madre e traviata, e che lo distendeva di speranza all'esempio del nonno. (ibid., p. 59)

Si imposta così, nella struttura profonda della narrazione, un rapporto allegorico tra la città e la madre, tra lo spazio relazionale pubblico e quello strettamente psicologico-affettivo :

La luce dorata del tramonto distendeva la città dentro le sue proporzioni, serena e solenne in ogni piazza e strada. Damìn fu colpito anche dalla somiglianza che trovava fervida tra ogni architettura, la sua luce e la sua bellezza, e il volto e la figura della madre : lo stesso portamento e lo stesso andare calmo della luce e dei gesti. La madre era bella e nobile come Urbino, come quella città piena di tempo e di storia eppure aperta e viva. Anche sulla città erano passati tiranni e prepotenti, e anch'essa era stata invasa e piegata a poteri contrari. Dovevano esserci ancora i luoghi e i segni delle loro violenze e distruzioni ; anche se quella luce avvolgeva tutto di uguale bellezza e continuava, caduto il sole, come se promanasse dalle stesse superfici che toccava.

Allo stesso modo camminava e guardava sua madre ; mentre la sua colpa continuava ad esistere dentro la sua bellezza, dentro le dimensioni composte della sua figura. (ibid., p. 165)

L'opera di Volponi acquista il significato di un rifiuto culturale e sociale del maschilismo e di un riconoscimento dei valori del femminile, inteso come luogo del profondo, dell'accoglienza, della fecondità :

Damìn era appena consolato dalla tiritera, formata soprattutto dalla invocazione " Matre maia » che poteva ripetere anche lui senza il timore e il dolore di pronunciare la parola madre, scaricandosi anzi con quella espressione estranea dei termini veri e incalzanti del proprio dramma. "Matre maia ", gli dava un verso ancora possibile, nuovo e anche dolce per riferirsi alla madre. (ibid., p. 52)

Il lanciatore di giavellotto è però anche la storia di un nucleo familiare in cui l'assenza del padre come modello è compensata dalla presenza del nonno (semanticamente rafforzata dall'omonimia tra avo e nipote), mentre la madre, figura privilegiata nel rapporto interpersonale con il figlio, è costretta a subire le attenzioni sessuali d'un gerarca fascista, che in questo modo impone schemi comportamentali autoritari e violenti coerenti con l'ideologia professata.

Il contesto che avvolge la vicenda familiare svolge una sua precisa funzione narrativa.

Le descrizioni della campagna marchigiana, le pagine in cui si esalta l'artigianato come espressione libera e completa della creatività dell'uomo, le riflessioni sulla necessità di trasformare ogni principio teorico in prassi, 
i riferimenti al valore della manualità come arte, sono tutti elementi che acquisiscono nella globalità del romanzo una dimensione ideologica.

A livello politico il romanzo è costruito sul contrasto tra il progressivo mutamento dell'eros - da forza ancestrale di vitalità a passione per la dignità dell'uomo, a progetto di lotta per un mondo più libero e più felice, ad ideologia rivoluzionaria -, e la rappresentazione allegorica della cultura fascista, oppressiva e violenta, che costringe il soggetto a rivolgere verso se stesso la violenza che lo opprime suicidandosi, ma che resterà sconfitta dalla forza utopica del gesto di rifiuto di Damìn e dalla lotta politica di Occhialini.

Attraverso una rete isotopica costituita da oggetti appuntiti, da simboli fallici aggressivi che rompono l'interiorità, la completezza sacra del corpo (il pugnale di Marcacci, la sciabola, il giavellotto, la roncola), Volponi denuncia la violenza maschile contro la dimensione femminile, il sopruso simbolico e fisico insito nell'atto stesso della penetrazione. E su questa simbologia complessa, di natura sessuale e ideologica, egli innesta il tema antropologico del sangue (in alcune parti dell'opera si ritrovano molte suggestioni del Pavese della Luna e i falò). Il sangue, secondo i codici rituali, deve rimanere interno, non può essere versato, proprio per la sua caratteristica mitica di elemento vitale dell'individuo. Se viene fatto fuoruscire, si commette peccato, si compie letteralmente un delitto, uno scelus.

C'è nel romanzo una sorta di macrosequenza che guida il lettore dalla scoperta del rapporto tra Marcacci e la madre di Damìn al suicidio del giovane che letteralmente si lancia da un ponte, si fa cioè egli stesso strumento di offesa e di morte.

Damin è colpito dal brillio del pugnale che pende dalla divisa di Marcacci mentre questi copula con sua madre e che egli identifica simbolicamente nel fallo del gerarca fascista che violenta la donna :

D'improvviso la donna alzò la faccia e la mostrò alla luna, bagnata dalla fronte al mento. Restò in quella posizione sotto la luce sempre più bianca ; mentre il nemico si riabbottonava i calzoni e si riaggiustava il pugnale : il manico di madreperla luccicava davanti e anche sulla bocca materna. (ibid., p. 34)

Egli stesso, educato ai rituali sportivi del fascismo, diventa lanciatore di giavellotto.

Ma poi, nel finale, incapace di tollerare il condizionamento di una società cosi intrisa di violenza e di ingiustizia, lancia la roncola contro la sorella, ne fa fuoruscire il sangue, e infine paga il suo delitto gettandosi nel vuoto dalla spalletta del ponte : 
Cercò anche di mettersi in equilibrio nell'impugnatura di se stesso proprio come un giavellotto, per andare a cadere quanto più possibile nel buio, dietro a Vitina, dietro il nastro del suo fiocco disfatto.

La visione istantanea quanto minuziosa del muretto che superava gli diede per un attimo un senso scuro di nostalgia per quel nastro, per il suo fiocco ancora composto, immagine di una verità dolce anche per lui, che aveva ormai infranto per sempre. Il nastro sciolto tracciava adesso nel buio il lancio di se stesso. La realtà del vuoto lo convinse del prodigio che infine giungeva a cogliere : senza più dolore né nostalgia ; con voluttà, con bravura. (ibid., p. 192)

Volponi ci propone in ultima analisi la storia di un processo di maturazione, psicologica e politica, favorito dal ruolo pedagogico di un personaggio come Occhialini, un semplice calzolaio, a cui è sottoposto un giovane che, nell'atmosfera ammorbata della dittatura fascista si ribella, con l'uccisione della sorella e il suicidio, al sopruso di una società che impone un modello del soggetto unidimensionale, emarginando qualunque forma di dissenso, di diversità.

Globalmente il lettore avverte che alla cultura della violenza fa da contraltare l'esaltazione della panspermia, della spinta alla generazione, della fecondità della natura, simbolizzata dall'organo femminile.

Si noti come, nel brano seguente, Occhialini svolga un preciso processo di educazione sessuale, tanto più funzionale alla coscienza del giovane Damìn, quanto più è incardinato nel contrasto tra la crudezza del linguaggio basso e popolare e il convinto rispetto della persona, della donna e della sua fisiologia del piacere :

- Ma è giusto che anche le donne debbano godere ?

- Eh sì, - aveva esclamato Occhialini, - anche le donne! Anche esse sono vive e libere e se volete figlie del signore... della terra, dell'amore, anch'esse provviste di un corpo e... persino del loro apposito organo per fare all'amore. Hanno tra le gambe la dolce, calda, bellissima fica, pelosa e aperta ; che non va mai rifiutata o nascosta e tanto meno ignorata : ma guardata, toccata, sfogliata labbro per labbro con le dita, con l'uccello e se si è già grandi, avanti con l'età e con l'esperienza, anche con il naso e con la lingua; specie se c'è l'amore vero, la simpatia e anche la pulizia. Questo faceva andare in estasi le francesi, che noi italiani non avevamo paura di guardargli la fica e anche di baciargliela e di leccargliela. O cos'è se no, una tomaia, una buca, una scarpa che s'infila ?... Perfino una scarpa deve essere apprezzata! Le donne debbono godere come gli uomini, e anche di più, per giustizia sociale e per compenso del partorire... La bravura di un uomo non è tanto nel come ce l'ha grosso e duro e nel numero delle volte che riesce a sbatterlo... ma proprio nell'aiutare e servire una donna ; nel farla godere, nel riconoscerle l'onestà di godere con il suo bel corpo... tutto, tutto il suo corpo.

Lo so, lo so che la borghesia conquista, usa e butta via ; ma questi appunto sono i padroni e i padroncini accecati dai pregiudizi, dai falsi orgogli e dalla Chiesa... questi sono i fascistacci sfruttatori, che riescono a fare all'amore solo con le puttane che sottomettono o nei casini, vere chiese della loro anima immatura e stravolta, della loro prepotenza animale... taurina, cornuta cioè, di veri cornuti. Ordunque, cari ragazzi, istruitevi per liberarvi e preoccupatevi sempre di guidare con simpatia la ragazza, di amarla bene, di farla godere con voi e di rispettarla dopo più di prima... e di ringraziarla con affetto, se ve l'ha data. (ibid., p. 26)

E'questo il manifesto di una utopia amorosa a cui s'accompagna quella politica. La speranza in un mondo migliore, offuscata dalla violenza del 
fascismo e della guerra, ritrova la sua strada nella scelta ideologica di Occhialini che, nel decidere di collaborare alla lotta per la liberazione, assume il nome di Damìn, per portare a termine quel compito politico e sociale a cui egli stesso lo aveva educato :

Avrebbe dovuto scegliere un nome convenzionale di lotta secondo le norme del partito comunista clandestino e comunicarlo al responsabile provinciale durante il prossimo incontro. Decise di prendere il nome di Damìn, in quel suo primo viaggio verso la rivoluzione : per affetto verso quello sciagurato giovane; ma anche perché sapeva che era caduto vittima della società borghese.

La sera lo colse dentro la campagna appoderata. Gli sembrò di sentire dei passi alle spalle, ma voltatosi non vide nessuno. Pensò che poteva essere seguito e sorvegliato e si fermò. Le colline intorno, ormai scure, risuonarono davvero di qualche colpo. La prima casa era sulla più alta e lontana, e luccicava con un bianco scialbo di calcina sui muri del pozzo. Quell'orizzonte soffice, smanato dalle grandi oscurità delle querce agli incroci e sui confini, lo intimidiva, lo spaventava anche con il ricordo di antiche magie e con l'incombenza di possibili agguati. Allora ripeté a se stesso di quali orizzonti, ben più cupi e smarginati, si fosse ormai circondata tutta la terra d'Europa, tutti percorsi e sconvolti dal fuoco della guerra; e come contro di essi venissero guidati eserciti sterminati e sterminatori e anche spinte intere popolazioni incatenate.

Riprese il passo verso la calcina bianca di quel pozzo lontano senza paura e senza più voltarsi. (ibid., p. 197-198)

\section{Il cammino era già tracciato.}

Nel 1989, con la pubblicazione di Le mosche del capitale, Volponi amplierà in prospettiva socio-economica la dialettica individuo-potere, già delineata nella filigrana tematica di Memoriale, e ci darà un affresco della civiltà industriale in cui le ombre, le oscurità trionfanti di un mondo cinico, feroce, che sembra aver perso, grottescamente, ogni segno di umanità, si alternano a permanenti tracce di speranza, a squarci di luce proiettati verso il futuro.

Quando, nel 1991, esce La strada per Roma, il romanzo progettato alla fine degli anni '50, iniziato nel '61, ripreso nel '64, e sempre interrotto, utilizzato, per testimonianza dello stesso autore, " come un deposito di materiale (emozioni e convinzioni) vero e utile ", si ha la conferma che la scrittura di Volponi, al di là dei mutamenti di struttura narrativa e poetica, al di là delle opzioni per temi apparentemente molto diversificati, presenta un tracciato circolare, un unico punto di partenza e di ritorno, individuabile nel continuo sforzo di immettere la voce dell'utopia nel silenzio di una realtà sempre più indecifrabile.

Il sogno della Città del Sole resta intatto nel mito di Urbino, il luogo in cui natura e storia trovano l'armonica compenetrazione, in cui è possibile riconoscersi e riconoscere, dunque interpretare : un modello aureo ma anche una testimonianza sulle possibilità che ha l'individuo, il singolo, di essere principio attivo della collettività, di farsi società : 
Urbino stava lassù, ignorata, isolata come un castello di ammalati. Chiunque saliva in piazza, cittadino o contadino, guardava il paesaggio e capiva ogni cosa, il giro del sole e il posto degli edifici, così come era contento di trovare il suo, all'ombra o al sole che fosse. Cittadino e villano, ciascuno parlava, contava gli anni e nominava le cose. Tutti finivano per guardare, in quella confusione tra il cielo e la terra, le colline alte, i vertici dei palazzi che restavano fuori, illuminati sotto il sole la cui luce non arrivava fino alle vallate. Erano fuori il Petralata, la torre della Brombolona, Crocicchia, San Cipriano, i Cappuccini e molto in fondo i sassi azzurri di San Simone. Una parentela minuta di chiese di mattoni, di querceti e di avellane, di campane di mezza voce, di magri pagliai. Dopo aver riconosciuti questi posti, tutti si accendevano le sigarette e cominciavano a camminare. Si avviavano verso il passeggio, in congregazioni ${ }^{9}$.

9. Id., La strada per Roma, Torino, Einaudi, 1991, p. 199. 